\title{
Fuzzy Filtering of Sensors Signals in Manufacturing Systems with Time Constraints
}

\author{
A. Mhalla, N. Jerbi, S. C. Dutilleul, E. Craye, M. Benrejeb
}

\author{
Anis M'halla, Nabil Jerbi, Mohamed Benrejeb \\ Ecole Nationale d'Ingénieurs de Tunis \\ Unité de recherche LARA-Automatique \\ BP 37, Le Belvédère, 1002 Tunis, Tunisie \\ E-mail: anis.mhalla@enim.rnu.tn,nabil.jerbi@isetso.rnu.tn,mohamed.benrejeb@enit.rnu.tn
}

Anis M'halla, Simon Collart Dutilleul, Etienne Craye ** Ecole Centrale de Lille

Laboratoire d'Automatique, Génie Informatique et Signal

Cité Scientifique, BP 48, 59651 Villeneuve d'Ascq, France

E-mail: simon.collart_dutilleul@ec-lille.fr, etienne.craye@ec-lille.fr,

\begin{abstract}
The presented work is dedicated to the supervision of manufacturing job-shops with time constraints. Such systems have a robustness property towards time disturbances. The main contribution of this paper is a fuzzy filtering approach of sensors signals integrating the robustness values. This new approach integrates a classic filtering mechanism of sensors signals and fuzzy logic techniques. The strengths of these both techniques are taken advantage of the avoidance of control freezing and the capability of fuzzy systems to deal with imprecise information by using fuzzy rules. Finally, to demonstrate the effectiveness and accuracy of this new approach, an example is depicted. The results show that the fuzzy approach allows keeping on producing, but in a degraded mode, while providing the guarantees of quality and safety based on expert knowledge integration.

Keywords: Alarm filtering, fuzzy logic, symptoms generation, robustness, time constraints, manufacturing.
\end{abstract}

\section{Introduction}

In general, the detection of failure symptoms related to the process elements requires a development of a system model to be supervised [1]. This model can be either a normal functioning model or dysfunction model. If a model is adopted, two basic mechanisms are used for detection. The first one consists of comparing the evolutions of the observed system with those of the process model, or with those of normal functioning signatures evolving in real time with the system. The second one is based on observing known failures signatures. These signatures describe historical or theoretical failures known from the process or the process elements. Without the supervision of a system model, the adopted strategy consists of an exploitation of the information given by the sensors and the detectors at a local level of the process [2]. Sometimes, in manufacturing workshops with time constraints, the information given by sensors signals is dubious and the symptoms generated are vague. Furthermore, the validation interval associated to each sensor signal is not always exact, this is the case where the temporal windows are badly defined. These reasons bring us to use fuzzy logic which is based on an approximate reasoning able to take into account the uncertainty and the inaccuracy of knowledge. This paper is an extension of Jerbi work [3]. In [3] was proposed an integration of the robustness in the filtering mechanism of sensors signals. This mechanism, presented by Toguyeni in 1992, aims at generating symptoms for the diagnosis [4]. Our main contribution is a fuzzy filtering mechanism of sensors signals. This paper is organised as follows. The first part summarizes the proposed filtering mechanism of sensors signals taking into account the robustness intervals. The second part introduces a new fuzzy filtering approach where 
fuzzy logic and filtering of sensors signals techniques are integrated. In order to show the effectiveness of this approach, in the third part, an illustrative example is outlined and the results are discussed. Finally, conclusions of this work are given.

\section{Robustness integration in the filtering of sensors signals}

\subsection{Symptoms generation}

Permanently, the state of the process model is updated by the evolutions caused by the control and the sensors signals. These sensors signals are sent by the controlled system in response to a control request. The mechanism developed for the detection of failures symptoms is based on the impact study of sensors signals on the process model, called reference model, and on that of the control. These two models do not make it possible to characterize all failures symptoms of a controlled system, for example, the absence of sensors signals (this can be the case, when a sensor is not functional or the control request was not carried out). In order to take into account these problems, mechanisms of "watchdogs" were integrated in the control and process models. These mechanisms are based on two dates provided by the scheduling task: the beginning date as soon as possible noted $\Delta t_{m / C R i}$ and the completion date noted $\Delta t_{M / C R i}$ of the control operation [4]. The idea consists of modelling any operation from a temporal approach. At each operation $A_{i}$ is associated a sensor signal $C R_{i}$. To each sensor signal $C R_{i}$ is associated a temporal interval $\left[\Delta t_{m / C R i}, \Delta t_{M / C R i}\right]$ (figure 1). The report $C R_{i}$ is valid only inside this window. $\Delta t_{m / C R i}$ and $\Delta t_{M / C R i}$ are defined relatively to the beginning of the operation $A_{i}$ (Start-Event). The filtering principle is to position the temporal window of each sensor signal $C R_{i}$ when its Start-Event was received.Two types of symptoms are distinguished:

- Symptoms type I noted $S_{i}^{1}$ : This class of symptoms corresponds to awaited sensor signal which is not received at $\Delta t_{M / C R i}$. The detection mechanism of this symptom type corresponds to the traditional mechanism of watchdog, but implemented in a separate way of the control.

- Symptoms type II noted $S_{i}^{2}$ : It is generated by the occurrence of a sensor signal which is not expected. Two cases are considered, the first one corresponds to an action but its report occurs before the validation interval. The second one corresponds to the occurrence of a report in absence of any control which can create it.

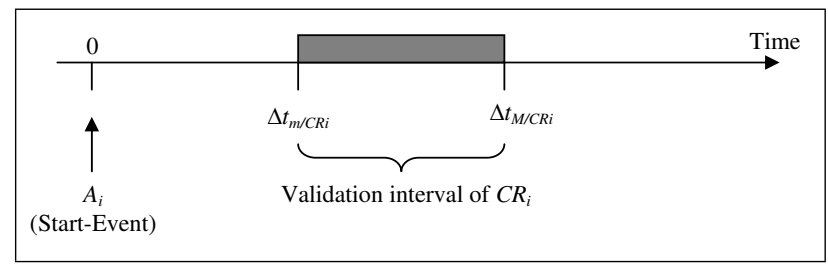

Figure 1: Operation associated model [4]

\subsection{Symptoms generation}

The robustness of a system can be defined as its ability to preserve the specifications facing some expected or unexpected variations. So, the robustness of a system characterizes its capacity to deal with disturbances [6]. It is interpreted into different specializations. The passive robustness is based upon variations included in validity time intervals. There is no control loop modification to preserve the required specifications. On the other hand, active robustness uses observed time disturbances to modify the control loop in order to satisfy these specifications. Therefore, the robustness intervals must be 
integrated in the filtering mechanism of sensors signals. In [3], a filtering mechanism of sensors signals integrating the robustness values is proposed. This mechanism allows the integration of the robustness results in the symptoms generation and the classification of the various actions reports. This classification is very useful for the supervision because it makes it possible to preserve the production function. This constitutes an enhancement of the filtering mechanism. Five time intervals, figure 2, are defined, namely: $I_{1 i}=\left[\Delta t_{m^{\prime \prime} / C R_{i}}, \Delta t_{m^{\prime} / C R_{i}}\left[I_{2 i}=\left[\Delta t_{m^{\prime} / C R_{i}}, \Delta t_{m / C R_{i}}\left[, I_{3 i}=\left[\Delta t_{m / C R_{i}}, \Delta t_{M / C R_{i}}\left[, I_{4 i}=\left[\Delta t_{M / C R_{i}}, \Delta t_{M^{\prime} / C R_{i}}[\right.\right.\right.\right.\right.\right.\right.$ and $I_{5 i}=\left[\Delta t_{M^{\prime} / C R_{i}}, \Delta t_{M^{\prime \prime} / C R_{i}}[\right.$.

The margin of passive robustness is available in $I_{2 i} \cup I_{4 i}$ whereas the margin of active robustness is in $I_{1 i} \cup I_{5 i}$. Several cases can arise:

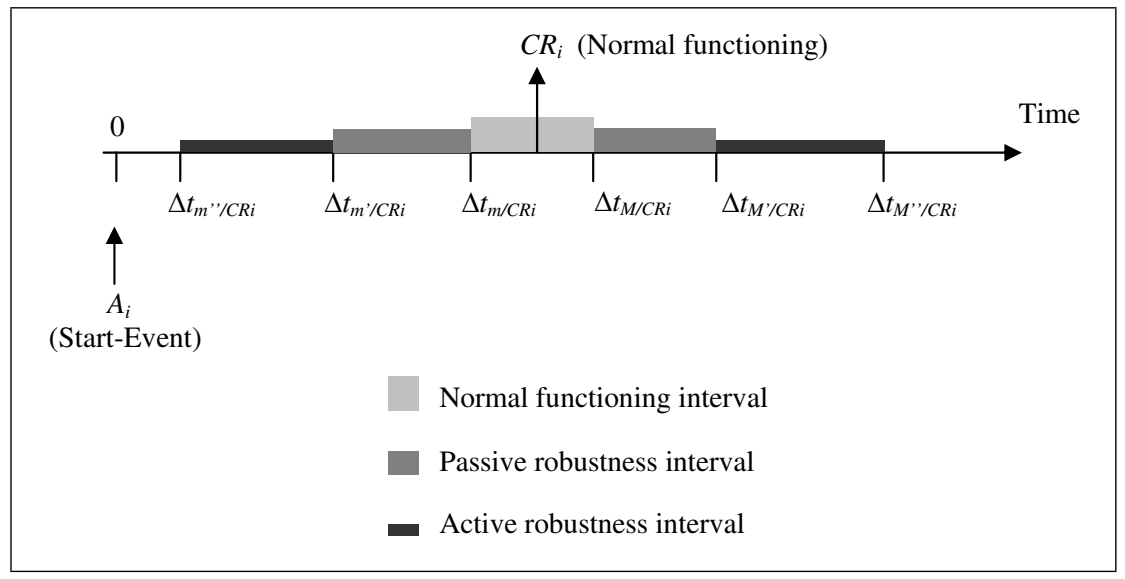

Figure 2: Robustness integration in the operation associated model [3]

- If there are absence of order (not $A_{i}$ ) and presence of $C R_{i}$, there are freezing of the control and generation of a symptom $S_{i}^{2}$.

- If the sensor signal $C R_{i}$ arrives in the time interval $\left[0, \Delta t_{m^{\prime \prime}} /{ }_{C R_{i}}\right.$, there are freezing of the control and generation of a symptom $S_{i}^{2}$.

- If the sensor signal $C R_{i}$ arrives in the time interval $I_{1 i}$, there are change of the control (active robustness to an advance) and memorizing a symptom $S_{i}^{2}$.

- If the sensor signal $C R_{i}$ arrives in the time interval $I_{2 i}$, there is no change of the control (passive robustness to an advance) but only a memorizing of a symptom $S_{i}^{2}$.

- If the sensor signal $C R_{i}$ arrives in the time interval $I_{3 i}$, the behavior of the system is normal.

- At the instant $\Delta t_{M / C R_{i}}$, there is automatically memorizing of a symptom $S_{i}^{2}$.

- If the sensor signal $C R_{i}$ arrives in the time interval $I_{4 i}$, it is a case of passive robustness to a delay. The symptom $S_{i}^{1}$ is already memorized.

- If the sensor signal $C R_{i}$ arrives in the time interval $I_{5 i}$, a change of the control is necessary (active robustness to a delay).

- At the instant $\Delta t_{M^{\prime \prime} / C R_{i}}$, there is freezing of the control.

Therefore, the robustness intervals are integrated in the filtering mechanism of sensors signals. It makes it possible to continue the production in a degraded mode. However the assumptions formulated in [3] are very restrictive. It is natural to consider different scenarios where the temporal specifications of the 
process are not fulfilled, nevertheless the production can continue. The next section presents a fuzzy filtering mechanism of sensors signals which introduces a finer classification of abnormal functioning and integrates the vague knowledge of the robustness intervals in the interpretation of sensors signals, coming from the workshop, for the generation of symptoms. The objective is to avoid the freezing of the control when the time disturbance is in the robustness intervals.

\section{$3 \quad$ Fuzzy filtering of sensors signals}

\subsection{Introduction}

Fuzzy logic is a mathematical tool that allows us to approach an unknown function by means of linguistic descriptions. Nevertheless, the linguistic information is a feature of the human reasoning and not of the mechanical components or programs. In consequence, this tool has achieved widespread applications and success in many areas such as control, supervision, image filtering and communications [6-9]. The essential characteristics of fuzzy logic are as follows [10]:

- Exact reasoning is viewed as a limiting case of approximate reasoning.

- Everything is a matter of degree.

- Any logical system can be fuzzified.

- Knowledge is interpreted as a collection of elastic or, equivalently, fuzzy constraint on a collection of variables.

- Inference is viewed as a process of propagation of elastic constraints.

Fuzzy logic calls upon a base of dubious knowledge, modelled by the sequence of fuzzy rules. This technique seems very promising thanks to its potential of use in dynamic monitoring and supervision, with the possibility of remaining human operator, by taking into account its way of reasoning and offering an interesting traceability. Before proceeding, we define some important terms.

Definition 1. [11]: A fuzzy set $F$ in a universe of discourse $U$ is characterized by a membership function $\mu_{F}: U \rightarrow[0,1]$

Definition 2. [11]: A linguistic variable $\mathrm{x}$ in a universe of discourse $\mathrm{U}$ is characterized by: $T(x)=$ $\left\{\mathrm{T}_{\mathrm{x}}^{1}, \mathrm{~T}_{\mathrm{x}}^{2}, \ldots, \mathrm{T}_{\mathrm{x}}^{\mathrm{k}}\right\}$ and $M(x)=\left\{\mathrm{M}_{\mathrm{x}}^{1}, \mathrm{M}_{\mathrm{x}}^{2}, \ldots, \mathrm{M}_{\mathrm{x}}^{\mathrm{k}}\right\}$ where $T(x)$ is the term set of $x$, that is the set of names of linguistic values of $x$ with each value $T_{x}^{i}$ being a fuzzy number with membership function $M_{x}^{i}$ on $U$.

\subsection{Basic Structure of a Fuzzy System}

Figure 3, shows the basic structure of a conventional fuzzy system. Such system can be seen as consisting of four basic building blocks: Fuzzification, Fuzzy rule set, Inference method and Defuzzification. Let us examine these building blocks in details:

- The fuzzification transforms a numerical input variable in a fuzzy set described by linguistic expressions.

- Fuzzy rules set: the fuzzy IF-THEN rule expresses a fuzzy implication relation between the fuzzy sets of the premise and the fuzzy sets of the conclusion.

- The inference makes it possible to implement, on the basis of fuzzy rules, the logical dependence between input variables and output fuzzy variables [12]. 


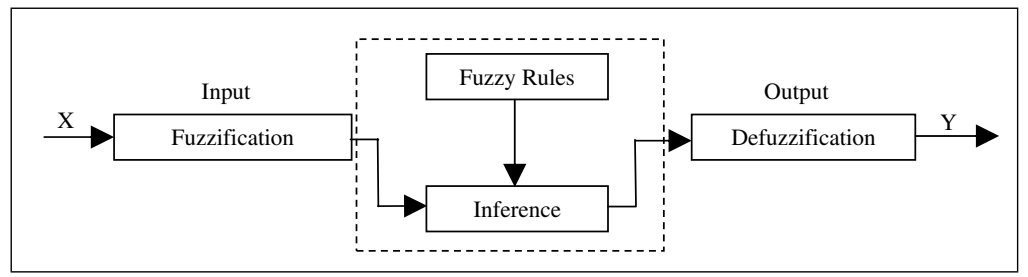

Figure 3: Fuzzy set stages

- The defuzzification transforms the output fuzzy set in a numerical variable.

Following the above definitions, the input vector $X$ which includes the input state linguistic variables $x_{i}$ 's, and the output state vector $Y$ which includes the output state linguistic variables $y_{i}$ 's, can be defined as:

$$
\begin{aligned}
& X=\left[x_{i}, U_{i},\left\{\mathrm{~T}_{\mathrm{x}_{\mathrm{i}}}^{1}, \mathrm{~T}_{\mathrm{x}_{\mathrm{i}}}^{2}, \ldots, \mathrm{T}_{\mathrm{x}_{\mathrm{i}}}^{\mathrm{k}}\right\},\left\{\mathrm{M}_{\mathrm{x}_{\mathrm{i}}}^{1}, \mathrm{M}_{\mathrm{x}_{\mathrm{i}}}^{2}, \ldots, \mathrm{M}_{\mathrm{x}_{\mathrm{i}}}^{\mathrm{k}}\right\}\right]_{i=[1, \ldots, n]} \\
& Y=\left[y_{i}, U_{i},\left\{\mathrm{~T}_{\mathrm{y}_{\mathrm{i}}}^{1}, \mathrm{~T}_{\mathrm{y}_{\mathrm{i}}}^{2}, \ldots, \mathrm{T}_{\mathrm{y}_{\mathrm{i}}}^{\mathrm{k}}\right\},\left\{\mathrm{M}_{\mathrm{y}_{\mathrm{i}}}^{1}, \mathrm{M}_{\mathrm{y}_{\mathrm{i}}}^{2}, \ldots, \mathrm{M}_{\mathrm{y}_{\mathrm{i}}}^{\mathrm{k}}\right\}\right]_{i=[1, \ldots, m]}
\end{aligned}
$$

The fuzzifier, in figure 3 , is a mapping from an observed input space to fuzzy sets in certain input universe of discourse. So, a specific value $x_{i}(t)$ at the time $\mathrm{t}$ is mapped to the fuzzy set $\mathrm{T}_{x}^{1}$ with degree $\mathrm{M}_{x}^{1}\left(x_{i}(t)\right)$ and to the fuzzy set $\mathrm{T}_{x}^{2}$ with degree $\mathbf{M}_{x}^{2}\left(x_{i}(t)\right)$ and so on.

\section{Fuzzification of the input and output variables}

If we want to introduce linguistic information, we have to define an interface. This interface is denominated fuzzification, and it translates the sensor measurements into linguistic concepts. To carry out such transformation, the fuzzification resorts to a characteristic function called membership function. The aim of fuzzification is to produce initial membership functions. Therefore, the universe of discourse $\mathrm{U}$ of the input and output variables are divided into fuzzy subsets.

The first step consists of choosing the input and output variables. This choice depends on the parameters available and the type of application [13]. In order to produce initial membership functions, the input and output spaces are divided into fuzzy regions. In our example, we have two inputs and two outputs variables, all membership functions are represented by trapezoidal forms. The sensor signal $\left(C R_{i}\right)$ and the occurrence of the Start-Event $\left(A_{i}\right)$ are considered as inputs variables. However, the type of symptom $\left(S_{i}\right)$ and the Control Decision (CD) are considered as outputs ones.

The second step consists of defining the universe of discourse which can take each variable. Then, we define the fuzzy sets associated to the inputs and outputs variables and their corresponding membership functions. Thus, the universe of discourse is divided into intervals at which a descriptive label is associated. This last choice is based on the experiment of the operator.

\section{- Fuzzification of sensor signal $\left(C R_{i}\right)$}

We define, figure 4, thirteen time intervals, namely: $I_{1 i}=\left[0, \Delta t_{m^{\prime \prime} / C R_{i}}\left[, I_{2 i}=\left[\Delta t_{m^{\prime \prime} / C R_{i}}, \Delta t_{m_{1}^{\prime \prime} / C R_{i}}\right.\right.\right.$, $I_{3 i}=\left[\Delta t_{m_{1}^{\prime \prime} / C R_{i}}, \Delta t_{m_{1}^{\prime} / C R_{i}}\left[, I_{4 i}=\left[\Delta t_{m_{1}^{\prime} / C R_{i}}, \Delta t_{m_{2}^{\prime} / C R_{i}}\left[I_{5 i}=\left[\Delta t_{m_{2}^{\prime} / C R_{i}}, \Delta t_{m_{1} / C R_{i}}\left[, I_{6 i}=\left[\Delta t_{m_{1} / C R_{i}}, \Delta t_{m_{2} / C R_{i}}[\right.\right.\right.\right.\right.\right.\right.$, $I_{7 i}=\left[\Delta t_{m_{2} / C R_{i}}, \quad \Delta t_{M_{1} / C R_{i}}\left[, \quad I_{8 i}=\left[\Delta t_{M_{1} / C R_{i}}, \quad \Delta t_{M_{2} / C R_{i}}\left[, \quad I_{9 i}=\left[\Delta t_{M_{2} / C R_{i}}, \quad \Delta t_{M_{1}^{\prime} / C R_{i}}\left[, \quad I_{10 i}=\right.\right.\right.\right.\right.\right.$ $\left[\Delta t_{M_{1}^{\prime} / C R_{i}}, \Delta t_{M_{2}^{\prime} / C R_{i}}\left[I_{11 i}=\left[\Delta t_{M_{2}^{\prime} / C R_{i}}, \Delta t_{M^{\prime \prime} / C R_{i}}\left[I_{12 i}=\left[\Delta t_{M^{\prime \prime} / C R_{i}}, \Delta t_{M_{1}^{\prime \prime} / C R_{i}}\left[\right.\right.\right.\right.\right.\right.$ and $I_{13 i}=\left[\Delta t_{M_{1}^{\prime \prime} / C R_{i}},+\infty[\right.$. The full set intervals is summarised in table 1 . 


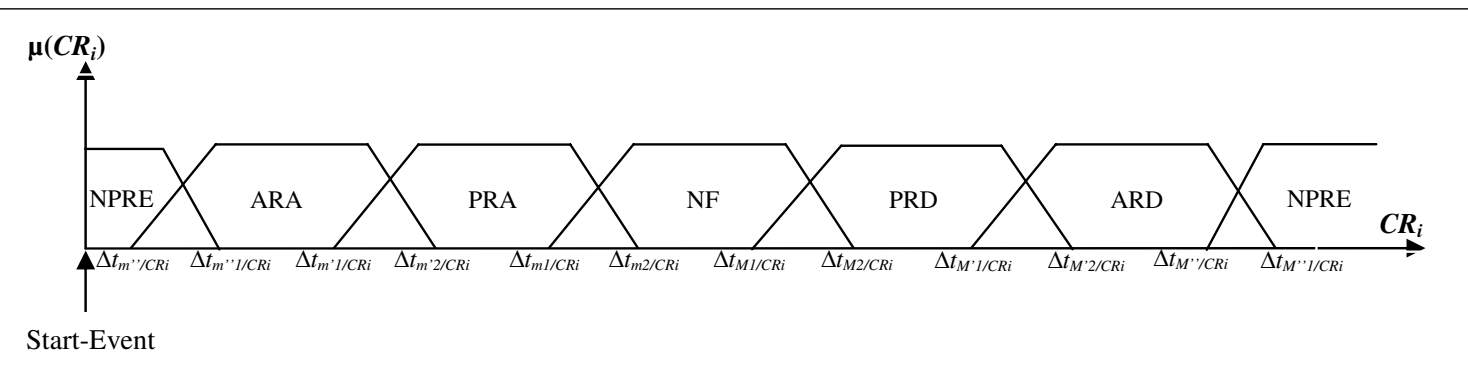

Figure 4: Fuzzy robustness integration in the operation associated model

Table 1: Linguistic variables associated to the input $C R_{i}$

\begin{tabular}{|c||c|}
\hline$T_{C R_{i}}$ & Linguistic variable \\
\hline \hline $\mathrm{T}_{C R_{i}}^{1}$ & $C R_{i}$ arrives in the interval $I_{1 i}=\left[0, \Delta t_{m^{\prime \prime} / C R_{i}}[\right.$ \\
\hline \hline $\mathrm{T}_{C R_{i}}^{2}$ & $C R_{i}$ arrives in the interval $I_{2 i}=\left[\Delta t_{m^{\prime \prime} / C R_{i}}, \Delta t_{m_{1}^{\prime \prime} / C R_{i}}[\right.$ \\
\hline \hline $\mathrm{T}_{C R_{i}}^{3}$ & $C R_{i}$ arrives in the interval $I_{3 i}=\left[\Delta t_{m_{1}^{\prime \prime} / C R_{i}}, \Delta t_{m_{1}^{\prime} / C R_{i}}[\right.$ \\
\hline \hline $\mathrm{T}_{C R_{i}}^{4}$ & $C R_{i}$ arrives in the interval $I_{4 i}=\left[\Delta t_{m_{1}^{\prime} / C R_{i}}, \Delta t_{m_{2}^{\prime} / C R_{i}}[\right.$ \\
\hline \hline $\mathrm{T}_{C R_{i}}^{5}$ & $C R_{i}$ arrives in the interval $I_{5 i}=\left[\Delta t_{m_{2}^{\prime} / C R_{i}}, \Delta t_{m_{1} / C R_{i}}[\right.$ \\
\hline \hline $\mathrm{T}_{C R_{i}}^{6}$ & $C R_{i}$ arrives in the interval $I_{6 i}=\left[\Delta t_{m_{1} / C R_{i}}, \Delta t_{m_{2} / C R_{i}}[\right.$ \\
\hline \hline $\mathrm{T}_{C R_{i}}^{7}$ & $C R_{i}$ arrives in the interval $I_{7 i}=\left[\Delta t_{m_{2} / C R_{i}}, \Delta t_{M_{1} / C R_{i}}[\right.$ \\
\hline \hline $\mathrm{T}_{C R_{i}}^{8}$ & $C R_{i}$ arrives in the interval $I_{8 i}=\left[\Delta t_{M_{1} / C R_{i}}, \Delta t_{M_{2} / C R_{i}}[\right.$ \\
\hline \hline $\mathrm{T}_{C R_{i}}^{9}$ & $C R_{i}$ arrives in the interval $I_{9 i}=\left[\Delta t_{M_{2} / C R_{i}}, \Delta t_{M_{1}^{\prime} / C R_{i}}[\right.$ \\
\hline \hline $\mathrm{T}_{C R_{i}}^{10}$ & $C R_{i}$ arrives in the interval $I_{10 i}=\left[\Delta t_{M_{1}^{\prime} / C R_{i}}, \Delta t_{M_{2}^{\prime} / C R_{i}}[\right.$ \\
\hline \hline $\mathrm{T}_{C R_{i}}^{11}$ & $C R_{i}$ arrives in the interval $I_{11 i}=\left[\Delta t_{M_{2}^{\prime} / C R_{i}}, \Delta t_{M^{\prime \prime} / C R_{i}}[\right.$ \\
\hline \hline $\mathrm{T}_{C R_{i}}^{12}$ & $C R_{i}$ arrives in the interval $I_{12 i}=\left[\Delta t_{M^{\prime \prime} / C R_{i}}, \Delta t_{M_{1}^{\prime \prime} / C R_{i}}[\right.$ \\
\hline \hline $\mathrm{T}_{C R_{i}}^{13}$ & $C R_{i}$ arrives in the interval $I_{13 i}=\left[\Delta t_{M_{1}^{\prime \prime} / C R_{i}},+\infty[\right.$ \\
\hline
\end{tabular}


The margin of active robustness is available in $\left(I_{2 i} \cup I_{3 i} \cup I_{4 i}\right) \cup\left(I_{10 i} \cup I_{11 i} \cup I_{12 i}\right)$, whereas the margin of passive robustness is in $\left(I_{4 i} \cup I_{5 i} \cup I_{6 i}\right) \cup\left(I_{8 i} \cup I_{9 i} \cup I_{10 i}\right)$. From a functional point of view, there are six intervals of use in which it is possible to prove the validity: intervals of No Proof of Robustness Existance (NPRE), Normal Functioning (NF), Passive Robustness to an Advance (PRA), Passive Robustness to a Delay (PRD), Active Robustness to an Advance (ARA) and Active Robustness to a Delay (ARD). If the functioning is abnormal, there is duality of advance and delay scenarios.

\section{- Fuzzification of a Start-Event $\left(A_{i}\right)$}

Figure 5, shows the different set of the input variable (Start-Event $A_{i}$ ). The full set of linguistic variables associated to each membership is summarised in table 2 .

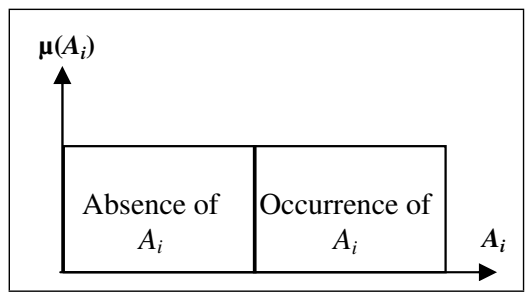

Figure 5: Membership functions of $A_{i}$

Table 2: Linguistic variables associated to the input Start - Event

\begin{tabular}{|c||c|}
\hline $\mathrm{T}_{A_{i}}$ & Linguistic variable \\
\hline \hline $\mathrm{T}_{A_{i}}^{1}$ & Occurrence of the Start-Event $A_{i}$ \\
\hline \hline $\mathrm{T}_{A_{i}}^{2}$ & Absence of the Start-Event $A_{i}$ \\
\hline
\end{tabular}

\section{- Symptoms fuzzification}

Figure 6, shows an uniform distribution of fuzzy logic membership functions associated to the output "type of symptom". Similarly, table 3 shows linguistic variables associated to the output " type of symptom ".

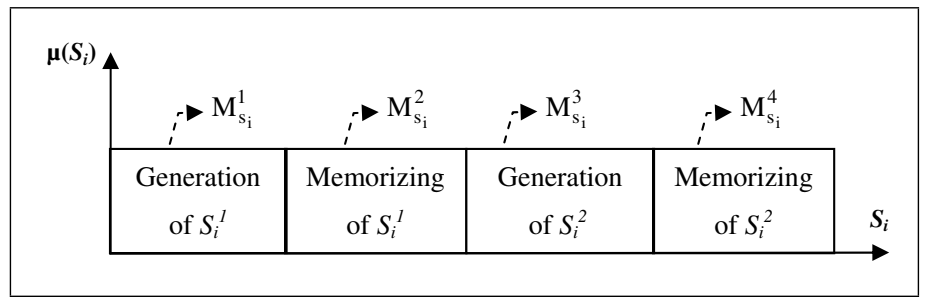

Figure 6: Membership functions of $S_{i}$ 
Table 3: Linguistic variables associated to the output " type of symptom"

\begin{tabular}{|c||c|}
\hline $\mathrm{T}_{S_{i}}$ & Linguistic variable \\
\hline \hline $\mathrm{T}_{S_{i}}^{1}$ & Generation of a symptom $S_{i}^{1}$ \\
\hline \hline $\mathrm{T}_{S_{i}}^{2}$ & Memorizing of a symptom $S_{i}^{1}$ \\
\hline \hline $\mathrm{T}_{S_{i}}^{3}$ & Generation of a symptom $S_{i}^{2}$ \\
\hline \hline $\mathrm{T}_{S_{i}}^{4}$ & Memorizing of a symptom $S_{i}^{2}$ \\
\hline
\end{tabular}

\section{- Fuzzification of Control Decision (CD)}

The three fuzzy set for the output $\mathrm{CD}$ are chosen as indicated in figure 7. Hence the three membership functions, uniformly distributed, are denoted $\mathrm{M}_{\mathrm{CD}}^{1}, \mathrm{M}_{\mathrm{CD}}^{2}$ and $\mathrm{M}_{\mathrm{CD}}^{3}$. The linguistic variables are summarised in table 4. The integration of the approach generation of symptoms and the classification of the reports of various actions allows a qualitative description of fuzzy variables. These variables have balanced values of truth, pertaining to the interval $[0,1]$.

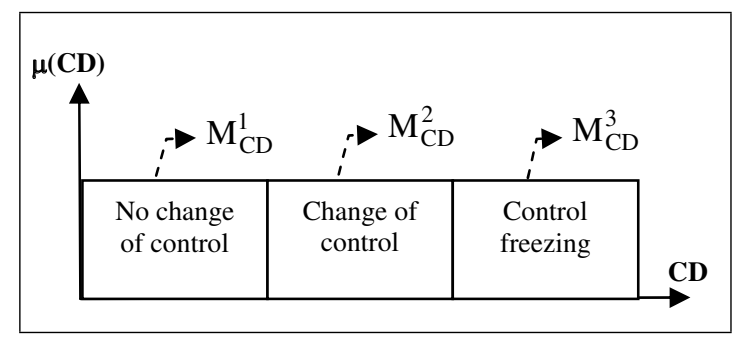

Figure 7: Membership functions of CD

Table 4: Linguistic variables associated to the output Control Decision

\begin{tabular}{|c||c|}
\hline $\mathrm{T}_{C D}$ & Linguistic variable \\
\hline \hline $\mathrm{T}_{C D}^{1}$ & No Change of control \\
\hline \hline $\mathrm{T}_{C D}^{2}$ & Change of control \\
\hline \hline $\mathrm{T}_{C D}^{3}$ & Control freezing \\
\hline
\end{tabular}

\section{Definition of fuzzy rules}

Next we have to evaluate the Rules. The rules associate the input variables with the output ones by means of linguistic terms, and according to their physical properties [14]. The rules can present different structures (MIMO, MISO, SISO, ...), although the most common one is the Multiple Inputs Multiple Outputs (MIMO). We have used this structure to build fuzzy rules, and its arrangement is:

$$
\begin{aligned}
& R_{M I M O}^{j}: \operatorname{IF}\left(x_{1} \text { is } \mathrm{T}_{x_{1}}\right) \text { AND } \ldots \text { AND }\left(x_{p} \text { is } \mathrm{T}_{x_{p}}\right) \\
& \operatorname{THEN}\left(y_{1} \text { is } \mathrm{T}_{y_{1}}\right) \text { AND } \ldots \text { AND }\left(y_{q} \text { is } \mathrm{T}_{y_{q}}\right) .
\end{aligned}
$$


Being $p$ the number of input variables, $q$ the number of output variables whereas $\mathrm{T}_{x_{i}}$ and $\mathrm{T}_{y_{i}}$ represent their respective fuzzy sets for $j^{\text {th }}$ rule.

The preconditions of $R_{M I M O}^{j}$, form a fuzzy set $\left(\mathrm{T}_{\mathrm{x}_{1}} \times \mathrm{T}_{\mathrm{x}_{2}} \times \ldots \times \mathrm{T}_{\mathrm{x}_{\mathrm{p}}}\right)$ and the consequent of $R_{M I M O}^{j}$ is the union of $q$ independent outputs [11]. So, the rule can be represented by a fuzzy implication:

$$
\mathrm{R}_{\text {мIмо }}^{\mathrm{j}}:\left(\mathrm{T}_{\mathrm{x}_{1}} \times \mathrm{T}_{\mathrm{x}_{2}} \times \ldots \times \mathrm{T}_{\mathrm{x}_{\mathrm{p}}}\right) \rightarrow\left(\mathrm{T}_{\mathrm{y}_{1}}+\ldots+\mathrm{T}_{\mathrm{y}_{\mathrm{q}}}\right)
$$

where "+" represents the union of independent variables. The fuzzy rules are merely a series of IF-THEN statements. These statements are usually derived by an expert to achieve optimum results. Thus, according to (3) we can formulate the rules as following:

Rule 1: IF there are absence of order (not $A_{i}$ ) AND presence of $C R_{i}$, THEN there are freezing of the control and generation of a symptom $S_{i}^{2}$.

Rule 2: IF the sensor signal $C R_{i}$ arrives in the time interval $\left[0, \Delta t_{m^{\prime \prime}} / C R_{i}\right.$ [ AND the Start-Event $A_{i}$ is occurred, THEN there are freezing of the control and generation of a symptom $S_{i}^{2}$.

Rule 3: IF the sensor signal $C R_{i}$ arrives in the time intervals $\left(I_{2 i} \cup I_{3 i} \cup I_{4 i}\right)$ AND the Start-Event $A_{i}$ is occurred, THEN there are change of control (ARA) and memorizing of a symptom $S_{i}^{2}$.

Rule 4: IF the sensor signal $C R_{i}$ report arrives in the time intervals $\left(I_{4 i} \cup I_{5 i} \cup I_{6 i}\right)$ AND the Start-Event $A_{i}$ is occurred, THEN there are no change of the control (PRA) and memorizing of a symptom $S_{i}^{2}$.

Rule 5: IF the sensor signal $C R_{i}$ arrives in the time intervals $\left(I_{6 i} \cup I_{7 i} \cup I_{8 i}\right)$ AND the Start-Event $A_{i}$ is occurred, THEN the behaviour of the system is normal (no change of the control).

Rule 6: IF the sensor signal $C R_{i}$ arrives in the time intervals $\left(I_{8 i} \cup I_{9 i} \cup I_{10 i}\right)$ AND the Start-Event $A_{i}$ is occurred, THEN it is the case of passive robustness to a delay (no change of the control) and memorizing of a symptom $S_{i}^{1}$.

Rule 7: IF the sensor signal $C R_{i}$ arrives in the time intervals $\left(I_{10 i} \cup I_{11 i} \cup I_{12 i}\right)$ AND the Start-Event $A_{i}$ is occurred, THEN a change of the control is necessary (ARD) and memorizing of a symptom $S_{i}^{1}$.

Rule 8: IF the sensor signal $C R_{i}$ arrives in the time interval $C R_{i}$ in the time interval $I_{13}$, THEN there are freezing of the control and memorizing of a symptom $S_{i}^{1}$.

Since the two outputs (Symptoms and Control Decision) of MIMO rule are independent, the general rule structure of MIMO fuzzy system can be represented as a collection of multiple-input and single-output (MISO) fuzzy systems by decomposing the above rules into $\mathrm{q}(\mathrm{q}=2)$ subrules with as the single consequent of the $j^{\text {th }}$ subrule. Therefore, the inference engine matches the rule preconditions in the fuzzy rule base with the input state linguistic terms and performs implication. In this subsection, for clarity, we will consider MISO system in the following analysis.

It is interesting to mention that each fuzzy rule just controls a part of the function to approach. These parts are denominated "patches", and they are the result of the localization property of the fuzzy basis functions. Nevertheless, from the fuzzy rules base (Rule 1, Rule 2, ..., Rule 8), we need to model numerically the operators AND and THEN. The fuzzy systems define an intermediate stage denominated Inference. The Inference is the part of the fuzzy systems that carries out an isomorphism between propositional logic and the Set and the Algebraic Theories. However, it is not valid whatever relation between the logical and math operators. In concrete, if they want to be equivalent, their logical and math tables for the crisp values $\{0,1\}$ have to be the same. The selected inference method is the Mamdani type which is known as the max-min method.

\section{Defuzzification}

After inferring all rules, the fuzzy systems need to fusion them.This is the main goal of the defuzzification step, and it constitutes the last part of all fuzzy systems. This fusion is not unique, although the Centre Of Area (COA) defuzzifier is the widespread one, figure 8. In the COA method, the fused 
measurement output $y^{*}$ is obtained as:

$$
y^{*}=\frac{\sum_{y \in Y} \mu_{F}(y) \cdot y}{\sum_{y \in Y} \mu_{F}(y)}
$$

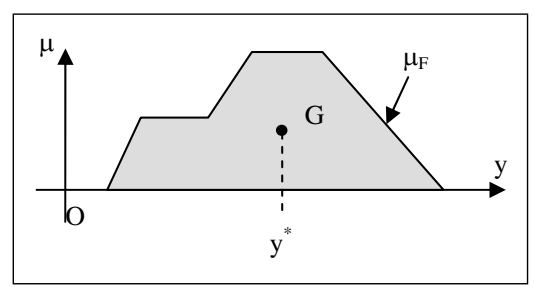

Figure 8: Centre Of Area defuzzifier

\section{Illustrative example}

To demonstrate the effectiveness and accuracy of the fuzzy filtering approach, an example with two fuzzy rules is outlined. Consider the following fuzzy rules base:

Rule 2: IF the sensor signal $C R_{i}$ arrives in the time interval $\left[0, \Delta t_{m^{\prime \prime}}{ }_{C R_{i}}\right.$ [ AND the Start-Event $A_{i}$ is occurred, THEN there are freezing of the control and generation of a symptom $S_{i}^{2}$.

Rule 3: IF the sensor signal $C R_{i}$ arrives in the time intervals $\left(I_{2 i} \cup I_{3 i} \cup I_{4 i}\right)$ AND the Start-Event $A_{i}$ is occurred, THEN there are change of control (ARA) and memorizing of a symptom $S_{i}^{2}$.

- Each rule use the operator "AND" in the premise, since it is an AND operation, the minimum criterion is used (Mamdani inference method), and the fuzzy outputs corresponding to these rules are represented by figure 9 and figure 10 .

- Next we perform defuzzification to convert our fuzzy outputs to a single number (crisp output), various defuzzification methods were explored to select the best one for this particular application. According to the relation(5), the weighted strengths of each output member function are multiplied by their respective output membership function center points and summed. Finally, this area is divided by the sum of the weighted member function strengths and the result is taken as the crisp outputs. In practice, there are two fuzzy outputs to defuzzify (Symptoms and Control Decision). To obtain a numerical output, we can take the COA of each fuzzy output, named $G_{S Y}$ and $G_{C D}$. The measures of the two CAO using specific values of sensor signal and Start-Event are summarized in table 5.

Table 5, shows the measures obtained by using defuzzification method mentioned above.Analysing the data, it is noted that the first and the third cases represent a classic filtering mechanism of sensors signals, integrating the robustness values described in [3]. The second case, using fuzzy filtering approach, gives better results than the two cases previously analysed. These cases reveal that the proposed approach is able to avoid control freezing (the COA $G_{C D}$ belongs to the membership function "change of control") same if the sensor signal arrives in the "No Proof of Robustness Existance (NPRE)" interval. Therefore, the fuzzy filtering approach makes it possible to continue the production in a degraded mode providing the guarantees of quality and safety. Consequently, the intelligent fuzzy logic control strategy, based on expert knowledge, provides the avoidance of control freezing if the time disturbance is in the robustness intervals. 


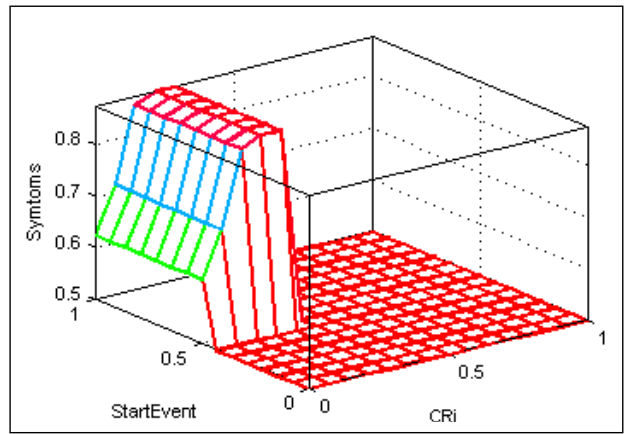

Figure 9: Three-dimensional trapezoidal membership function: Symptoms $=f\left(C r_{i}, A_{i}\right)$

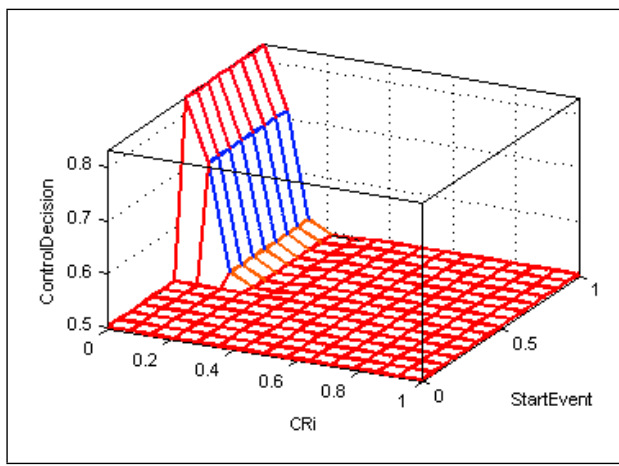

Figure 10: Three-dimensional trapezoidal membership function: Control Decision $=f\left(\mathrm{Cr}_{i}, A_{i}\right)$

Table 5: Measures of $G_{S Y}$ and $G_{C D}$

\begin{tabular}{|c||c||c||c|}
\hline Variables & & Measures & \\
\hline \hline & First Case & Second Case & Third case \\
\hline \hline$C R_{i}$ & $\begin{array}{c}C R_{i} \text { arrives } \\
\text { in the interval } I_{1 i}\end{array}$ & $\begin{array}{c}C R_{i} \text { arrives } \\
\text { in the interval } I_{2 i}\end{array}$ & $\begin{array}{c}C R_{i} \text { arrives } \\
\text { in the interval } I_{3 i}\end{array}$ \\
\hline \hline$A_{i}$ & $A_{i}$ is occurred & $A_{i}$ is occurred & $A_{i}$ is occurred \\
\hline \hline$G_{C D}$ & $G_{C D} \in M_{C D}^{3}$ & $G_{C D} \in M_{C D}^{2}$ & $G_{C D} \in M_{C D}^{1}$ \\
\hline \hline$G_{C D}$ & $G_{C D} \in M_{S i}^{3}$ & $G_{C D} \in M_{S i}^{4}$ & $G_{C D} \in M_{S i}^{4}$ \\
\hline
\end{tabular}

\section{Conclusion}

This paper deals with supervision of manufacturing workshops with time constraints. A new approach integrating a classic filtering mechanism of sensors signals and fuzzy logic techniques has been presented. This approach exploits the advantages that both techniques have: the avoidance of control freezing using robustness knowledge and the ability of fuzzy systems to deal with imprecise information by using fuzzy rules.

In this new approach, an enhancement technique based on various combinations of fuzzy logic linguistic statements in the form of IF-THEN rules, based on expert knowledge, makes it possible to continue the production in a degraded mode providing the guarantees of quality and safety.The establishment of fuzzy logic is interesting, but it is necessary to call upon the human expertise, in an environment of uncertainty and imprecision, able to formulate and to transmit its knowledge for decision making. 
The results obtained in the illustrative example show that this fuzzy approach is effective in situations where the sensor measurement is contaminated with different kind of noises. In this case, the temporal windows associates to each sensor signal are badly defined.

Conventionally, the selection of fuzzy IF-THEN rules often relies on a substantial amount of heuristic observation to express proper strategy's knowledge. Obviously, it is difficult for human experts to examine all the input-output data from a complex system to find the suitable number of rules within the fuzzy systems. For this reason, a fuzzy system with neural network's learning ability is required. A new approach using Neural Fuzzy Filter (NFF), based upon a neural network's learning ability and fuzzy IF-THEN rule structure can be developed in order to supervise critical time manufacturing job-shops. This fuzzy filtering approach shows how the knowledge of the robustness could make the supervision more efficient, by introducing two events (Start-Event and sensor signal). A chronicle recognition approach, using the additional information provided by the occurrences of intermediate events, is a challenging technique for performing early diagnosis.

\section{Bibliography}

[1] A. Boufaied, A. Subias, and M. Combacau, Distributed Fault Detection with Delays Consideration, $15^{\text {th }}$ International Workshop on Principles of Diagnosis, Carcassonne, June 2004.

[2] A. Boufaied, A. Subias, and M. Combacau, The Distributed time constraints verification modelled with time Petri nets, $17^{\text {th }}$ IMACS Word Congress on Scientific Computation, Applied Mathematics and Simulation (IMACS’05), Paris, July 2005.

[3] N. Jerbi, S. Collart Dutilleul, E. Craye, and M. Benrejeb, Time Disturbances and Filtering of Sensors Signals in Tolerant Multi-product Job-shops with Time Constraints, International Journal of Computers, Communications \& Control, Vol. 1, No. 4, pp. 61 - 72, 2006.

[4] A. Toguyeni, Surveillance et diagnostic en ligne dans les ateliers flexibles de l'industrie manufacturière, Ph.D. Thesis, Université des Sciences et Technologies de Lille, November 1992.

[5] N. Jerbi, S. Collart Dutilleul, E. Craye, and M. Benrejeb, Robust Control of Multiproduct Job-shops in Repetitive Functioning Mode, IEEE Conference on Systems, Man, and Cybernetics (SMC'04), The Hague, Vol. 5, pp. 4917 - 4922, October 2004.

[6] N. Sawaya, and B. Ghaddar, A Fuzzy Logic Approach for Adjusting the Contention Window Size in IEEE 802.11e Wireless Ad hoc Networks, IEEE International Symposium on Communication, Control, and Signal Processing (IEEE ISCCSP), Marrakech 2006.

[7] J. Bas, A. Pérez, and M. Lagunas, Differential fuzzy filtering for adaptive line enhancement in spread spectrum communications, Signal Processing Journal, Vol. 86, Issue 5, pp 984 - 1009, May 2006.

[8] D. Van De Ville, M. Nachtegael, D. Van der Weken, E. Kerre, and W. Philips, Noise Reduction by Fuzzy Image Filtering, IEEE Transactions on Fuzzy System, Vol. 11, No. 4, pp. 429 - 436, August 2003.

[9] R. Mikut, A. Lehmann, and G. Bretthauer, Fuzzy Stability Supervision of Robot Grippers, IEEE International Conference on Fuzzy Systems, Budapest, Vol. 3, pp. 1473 - 1478, July 2004.

[10] L. Zadeh, Knowledge Representation in Fuzzy Logic, IEEE Transactions on Knowledge And Data Engineering, Vol. 1, No. 1, pp. 89 - 100, March 1989.

[11] C. Teng Lin, An Adaptive Neural Fuzzy Filter and Its Applications, IEEE Transactions on Systems Man and Cybernetics, Vol. 27, No. 4, pp. 635 - 656, August 1997. 
[12] F. Lotte, A. L'ecuyer, F. Lamarche, and B. Arnaldi, Studying the Use of Fuzzy Inference Systems for Motor Imagery Classification, IEEE Transactions on Neural Systems and Rehabilitation Engineering, Vol. 15, No. 2, June 2007.

[13] Z. Shafiq, F. Muddassar, and S. Khayam, A Comparative Study of Fuzzy Inference Systems, Neural Networks and Adaptive Neuro Fuzzy Inference Systems for Portscan Detection (EvoWorkshop), pp. $52-61,2008$.

[14] J.L. Castro, J.M. Benitez, and I. Requena, Are artificial neural networks black boxes?, IEEE Transaction on Neural Networks, Vol. 8, No. 5, pp.1156 - 1164, September 1997.

Anis M'halla was born in Mahdia, Tunisia in 1980. He obtained the Engineer degree in electroMechnical engineering from the "Ecole Nationale d'Ingénieurs de Sfax (ENIS) " and obtain the master degree in automatic and industrial Maintenance from the "Ecole Nationale d'Ingénieur de Monastir" in 2006. He is currently preparing the Ph.D. degree in automatic and computer science within the framework of LAGIS-EC-Lille and LARA-ENIT cooperation. His research is related to robustness and supervision of multi-product job-shops with time constraints.

Nabil Jerbi was born in Tunis, Tunisia, in 1970. He obtained the Engineer degree in electrical engineering from the Ecole Nationale d'Ingénieurs de Tunis (ENIT), in 1994. Also, he received the Aggregation Certificate from the Ecole Supérieure des Sciences et Techniques de Tunis and the Master degree in automatic and signal treatment from ENIT, in 2001 and 2003, respectively. $\mathrm{He}$ is currently an assistant at "Institut supérieur des Sciences Appliquées et de Technologie de Kairouan ". His research interests include robustness and supervision of multi-product job-shops with time constraints.

Pr. Simon Collart Dutilleul obtained a Ph.D. degree in Electronics, Electrotechnics and Automatic Control from Université de Savoie in 1997. He is currently a Professor at Ecole Centrale de Lille, and a member of the research team on Discrete Event Systems in the LAGIS laboratory (Laboratory of Control Engineering, Computer Science \& Signal), where he studies specifically time constrained systems.

Pr. Etienne Craye was born in Roubaix (France) in 1961; he obtained in 1984 the Engineer Diploma of the "Institut Industriel du Nord" (French "Grande Ecole") and the same year his Master Degree in Computer Sciences. He obtained a Ph.D. in Automatic control for Manufacturing and Discrete Events systems in 1989 and his "Habilitation ŕ Diriger des Recherches" in 1994. He is now, since 1995, Professor at the Ecole Centrale de Lille and in the same time the Director of this institution. Pr. Craye is currently working on Monitoring and Supervision of Fault Tolerant Systems. Specially, reconfiguration and working mode management are today studied by taken into account on one-hand failures and on the other hand the flexibilities of the system architecture. The objective is to be able to go on with the production and not to reconsider the objectives.

Pr. Mohamed Benrejeb was born in Tunisia in 1950. He obtained the Diploma of "Ingénieur IDN" (French "Grande Ecole") in 1973, The Master degree of Automatic Control in 1974, the $\mathrm{PhD}$ in Automatic Control of the University of Lille in 1976 and the DSc of the same University in 1980. Full Professor at "Ecole Nationale d'Ingénieurs de Tunis" since 1985 and at "Ecole Centrale de Lille" since 2003, his research interests are in the area of analysis and synthesis of complex systems based on classical and non conventional approaches. 